desde la academia

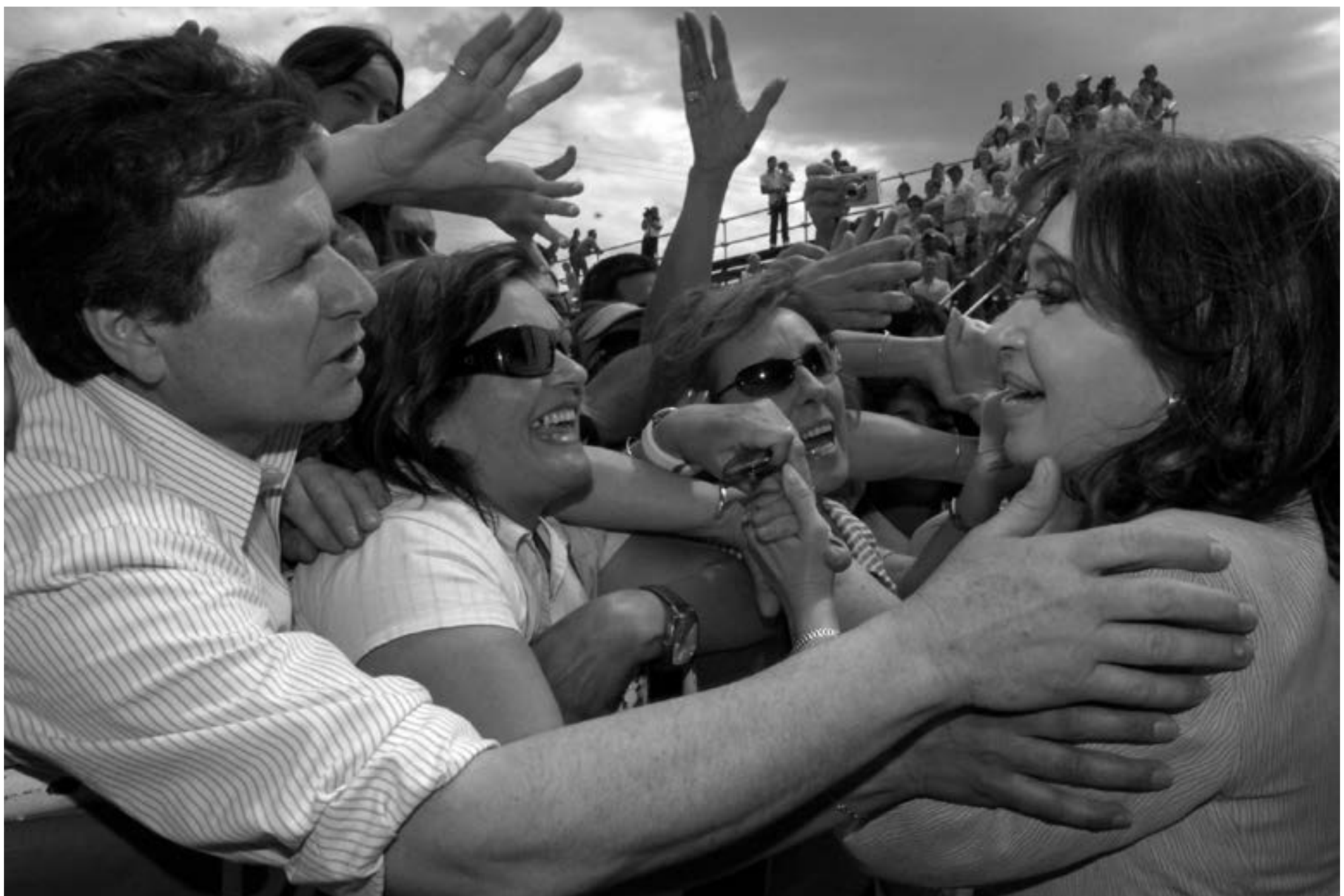

Foto: ${ }^{\oplus A F P} /$ Presidencia de Argentina. Cristina Fernández, entonces candidata presidencial argentina, saluda a sus seguidores en un acto. Buenos Aires, octubre de 2007

\title{
Esperanza y miedo: una aproximación teórica a las emociones en la comunicación política
}

Hope and fear: a theoretical approach to the emotions in political communication

https://doi.org/10.22235/d.v0i25.1271

Raquel Tarullo 


\section{RESUMEN}

Los postulados de la filosofía occidental presentan a la razón y la emoción como dos fuerzas antagónicas. En Occidente, la razón ha tenido que imponerse por sobre la emoción para que las personas tomaran sus decisiones objetivamente. El planteo de Habermas sobre la dimensión racional de la esfera pública reafirma esta tendencia, al considerar que el dominio de lo público debe buscar un orden basado en principios racionales. Si bien en las investigaciones en ciencias sociales el racionalismo continúa predominando, se evidencia un giro en los abordajes, que incorporan el ámbito emocional para comprender fenómenos contemporáneos. Particularmente en la comunicación política, la aproximación a las emociones como objeto de estudio es aún incipiente. Sin embargo, nuevas perspectivas disciplinares permiten innovar en las líneas de investigación en este campo. El objetivo de este artículo es realizar una revisión bibliográfica de los principales postulados de la teoría de la inteligencia afectiva que, de la mano de la neurociencia, aportan otras miradas a los procesos de comunicación política.

Palabras clave: emoción, razón, comunicación política, inteligencia afectiva, redes sociales

\section{Introducción ${ }^{1}$}

El arribo de las emociones al campo de la comunicación en general, y al de la comunicación política en particular, viene de la mano de los cambios socioculturales propios de la época. La vida pública comienza a desdibujar sus límites y lo que antes pertenecía a la esfera de lo privado adquiere una dimensión que parece ir conquistando todos los espacios. Aquello que sustenta la vida del sujeto cívico, que lo conmueve, que lo emociona, sus cuestiones personales y privadas, dejan de ser parte del santuario de la intimidad para entrar en el terreno de lo político y lo público (Dahlgren, 2012). Asuntos como la orientación sexual, la familia, el aborto y el cuerpo se convierten en temas de

\section{ABSTRACT}

The Western philosophy postulates present reason and emotion as antagonistic forces that motivate individuals in their everyday decision making. In the Western culture, reason has had to dominate emotion so as to reach objective decisions. Habermas hypothesis about the rational dimension of the public sphere reaffirms this tendency that states the public domain should find an order based on rational principles. Although rationalism is still predominant among Social Science researchers, a change in the approaches is evident: emotions have begun to be included in studies when the comprehension of contemporary phenomenon is necessary. Particularly, in the political communication field, emotion as a topic of exploration is new. However, new disciplinary perspectives allow innovating in research inquiries. The aim of this article is to examine literature about the main postulates of the Affective Intelligence Theory which, with Neuroscience, offer other perspectives to the political communication field.

Keywords: emotion, reason, political communication, affective intelligence, social media

la política y abandonan su privacidad. De esta perspectiva proviene la denominación ciudadano intimo (Plummer, 2003) para definir un escenario en el que las cuestiones íntimas -que tanto tienen que ver con la emoción y el afecto- se vuelven públicas y llegan incluso a ser sustento de políticas públicas.

Asimismo, la fragmentación de la audiencia, la proliferación de canales de comunicación y la expansión de los medios de comunicación personales han cambiado la forma en que los dirigentes politicos se comunican con los ciudadanos: es una etapa novedosa en la que tienen que responder y satisfacer a los medios y al público (Blumler y Gurevitch, 1995; Blumler y Kavanagh, 1999).
Raquel Tarullo

Universidad Nacional del Noroeste de la Provincia de Buenos Aires Buenos Aires, Argentina raqueltartullo@gmail.com

Recepción: mayo 2016 Aceptación: setiembre 2016
$1::$ Este artículo se inscribe en una investigación más amplia, que constituye una tesis de doctorado que estudia cómo comunican los dirigentes políticos argentinos en Facebook. 
De esta manera, los dirigentes políticos comienzan a adaptar sus mensajes a los tiempos que corren y dejan de lado las cuestiones cívicas, las políticas públicas y todo aquello que precise de mayor atención y concentración: sus mensajes se encuentran a la misma hora y en el mismo canal que las estrellas de espectáculo, que parecen decir cosas que despiertan más interés en la audiencia que las promesas de campaña (Rincón y Bonilla, 2004; Rincón, 2011).

En este escenario el discurso político ya no es dominante, por lo que intenta encontrar su lugar en el abanico de posibilidades que brindan las manifestaciones de la cultura popular (Bennett, 1998; Rincón y Bonilla, 2004) y deja a un costado al debate racional, la argumentación y la búsqueda de consensos (Blumler y Gurevitch, 1995; Wolton, 2005).

A pesar de estos cambios, en el marco de la comunicación política el plano emocional no ha sido estudiado en profundidad. En la mayoría de las investigaciones, las emociones de los actores incluidos en el proceso comunicacional ni siquiera han sido tenidas en cuenta (Valenzuela, 2011). Esta falencia dentro del campo disciplinar se debe no tanto a la negación de las emociones como objeto de estudio, sino al insistente uso de aproximaciones teóricas que responden a paradigmas donde prima la razón (Bennett e Iyengar, 2008).

Valenzuela (2011) afirma que los tres paradigmas más conocidos en los estudios sobre los efectos de los medios realizan sus abordajes con especial énfasis en aspectos cognitivos tales como la atención, la elaboración y el conocimiento (Chaffee y Schleuder, 1986; Eveland, 2004). Estos paradigmas son la teoría de la agenda-setting (López-Escobar y Llamas, 1996; McCombs y Shaw, 1974), el priming (Iyengar y Kinder, 1987) y el framing (Reese, 2001). A estos se les agrega el modelo sobre la comunicación en dos pasos -que sirvió de sustento a posteriores modelos sobre comunicación pública y ciudadana (Katz y Lazarsfeld, 1955)- y los más recientes estudios sobre deliberación democrática (Moy y Gastil, 2006).

Otros trabajos (Banning y Coleman, 2009; Coleman y $\mathrm{Wu}, 2010$; Miller, 2007) han integrado las emociones al estudio de la comunicación política al sostener que estas son centrales a la experiencia humana y que, por ello, no pueden quedar afuera del análisis de la comunicación de los gobiernos y los políticos con los ciudadanos (Dang-Xuan, Stieglitz, Wladarsch y Neuberger, 2013; Stieglitz y Dang-Xuan, 2013). En el caso de la teoría de agenda-setting, por ejemplo, se han incluido aspectos relacionados con los afectos - como la valencia, la relevancia y una categorización de las emociones como positivas o negativas-, pero solo como auxiliares de un proceso donde prima la cognición (Valenzuela, 2011).

En este contexto de tironeo entre razón y emoción, de críticos que entienden que una prevalece sobre la otra, algunos autores - muchos de ellos provenientes de la teoria de la inteligencia afectiva- entienden que racionalidad y emoción están enlazadas, y que ambas forman parte del individuo como dimensiones imprescindibles para que este se desarrolle como sujeto social.

La persona es capaz de ser racional porque es emocional. Las emociones activan la razón. La práctica de la ciudadanía debe admitir el rol que cumple la emoción en el desarrollo de la racionalidad, el esfuerzo por eludir a la pasión también socava la capacidad de razonar (Marcus, 2002, p.7).

Distintas voces académicas insisten que en la comunicación política $-\mathrm{y}$ aunque la literatura sobre la dimensión inconsciente del sujeto cívico sea escasa- es preciso no solo prestar atención a aquello que está en relación con el argumento, el debate formal y la información: hay que atender también a los símbolos, la retórica, la imagen, 
las alegorías, las emociones y todo aquello que está por detrás, o al costado, de la razón. El sujeto ciudadano, el sujeto civil, no es solamente razón, es también emoción, y una dimensión no puede sobrevivir sin la otra (Brader, 2005; Dahlgren, 2012; Marcus, 2002; Russell, Marcus, Crigler y Mackuen, 2007; Valenzuela, 2011).

Las emociones también están en el cerebro

$\mathrm{Si}$ "la comunicación se produce activando las mentes para compartir significado" (Castells, 2008, p. 191), entender qué es aquello que activa las mentes pondría claridad al análisis de los procesos comunicacionales.

Las emociones han sido ampliamente abordadas desde la psicología, campo disciplinar en el que se origina su estudio. Un ejemplo es el acercamiento funcional al concepto de las emociones desarrollado Klaus Scherer (2005). También desde la psicología se han aportado iluminadores hallazgos al campo de la comunicación política, tales como los trabajos de Jennifer Jerit (2004) y de Agustín Espinosa Pezzia (2008) sobre la incidencia de las emociones en las elecciones generales en Perú en 2006, entre otros.

En los últimos años, la teoría de la inteligencia afectiva profundiza el rol de las emociones en la comunicación política y se nutre, fundamentalmente, de aportes de la neurociencia. Antonio Damasio ha dedicado gran parte de sus investigaciones al estudio de la emoción como motor del pensamiento y comportamiento. Este autor rompe con la antigua dicotomía entre razón y emoción al ubicarlas a ambas en el cerebro, donde comparten espacios, redes neuronales y decisiones (Damasio, 1994).

Damasio retoma la línea filosófica de Spinoza, filósofo holandés que en la segunda mitad del siglo VXII afirmaba, en disonancia con el racionalismo imperante, que "los impulsos, motivaciones, emociones y sentimientos (un conjunto que él denominaba afectos) eran un aspecto fundamental de la humanidad" (Damasio, 2007, p. 15).
Manuel Castells dedica un capítulo de su libro Comunicación y poder (2009) a explicar los estudios de Damasio, a partir de conversaciones mantenidas con el científico. Allí se establece que la construcción de la realidad está dada por el procesamiento inconsciente que el cerebro hace de los acontecimientos, tanto internos como externos, pero con el uso de sus propios modelos para dar lugar a nuevas imágenes mentales. Damasio, dice Castells, investiga el rol de las emociones en estos procesos y su influencia en el comportamiento social.

Las emociones son modelos diferenciados de respuestas químicas y neuronales al detectar el cerebro un estímulo emocionalmente competente (ECS), es decir, cambios en el cerebro y en el propio organismo inducidos por el contenido de una percepción (tales como un sentimiento de miedo cuando vemos una imagen que nos recuerda la muerte) (Castells, 2008, p. 195).

Los conceptos desarrollados por George Lakoff también dan sustento al marco de análisis de la comunicación política. Lakoff sostiene que las metáforas son las estructuras físicas del cerebro que habilitan sus procesos de pensamiento para entender conceptos y realizar razonamientos abstractos. La metáfora se activa con el lenguaje, a partir del recurso narrativo (Lakoff, 2007). En este sentido, el lenguaje cumple un rol fundamental en la activación de los marcos conceptuales, que dan cuenta de las percepciones que cada persona tiene de la realidad.

Los marcos no se ven, no se oyen. Forman parte del inconsciente cognitivo y solo podemos acceder a ellos de modo indirecto a través de sus consecuencias y del lenguaje. Todas las palabras adquieren su sentido dentro de un marco (Gutiérrez-Rubí, 2009, p. 25). 
Los marcos están inscriptos en el cerebro, en los circuitos neuronales: la decisión de una persona sobre qué hacer con la información recibida depende, en gran medida, de la coherencia de esta información con los marcos configurados previamente.

La clave es cómo activar esos valores latentes, cómo hacer que el deseo de solidaridad sea más fuerte que la agresividad individualista o el deseo de paz más fuerte que el miedo (Castells citado en Gutiérrez-Rubí, 2009, p. 26).

Es en la mente donde las emociones y los sentimientos se conectan para influir en la toma de decisiones. En este proceso de conexiones entran en juego las redes de asociación con las que cuenta la mente, los acontecimientos internos y externos, los acontecimientos previsibles y los mapas del cerebro (Castells, 2008). Para que el cerebro conecte y relacione los mapas con los acontecimientos externos debe producirse un acto de comunicación, es decir, se precisa del lenguaje para que la mente se active y se compartan significados, que es el fin de la comunicación (Castells, 2008, 2012).

Dos sistemas para activar las emociones "El cerebro político es un cerebro emocional", afirma Westen (2008, p. 4) en una sentencia que marca profundamente el campo disciplinar de la comunicación política. La teoría de la inteligencia afectiva sostiene que las decisiones políticas se toman mediante una integración entre la razón y la emoción. La cuestión no es en cuál de las dos prevalece la toma de decisiones, sino cómo se procesa la información a partir del objeto sobre el que es preciso tomar una decisión. Siguiendo esta línea, la persona puede decidir basada solo en la información recibida (cognición) o completarla con experiencias pasadas constituidas en marcos conceptuales (Castells, 2009; Lakoff, 2007).
Desde la comunicación y la psicología política, la teoría de la inteligencia afectiva brinda herramientas teóricas para entender los procesos de toma de decisiones y la importancia que tienen tanto la emoción como la razón al funcionar de manera complementaria. Esta corriente analítica tiene implicaciones importantes para el estudio del comportamiento político, especialmente el comportamiento electoral (Brader, 2005; Marcus, 2002; Russell et al., 2007).

La teoria de la inteligencia afectiva afirma que, lejos de obstaculizar el razonamiento de las personas, las emociones colaboran en la relación que estas establecen con el contexto. Así, la razón y la emoción no compiten en el proceso de la toma de decisiones: la emoción no nubla a la razón, sino que la complementa (Damasio, 1994; LeDoux, 1996). Este concepto, en el marco de la comunicación política, aporta sólidas líneas de análisis para investigar cómo comunican los dirigentes políticos (Brader, 2005).

De acuerdo con la teoría de la inteligencia afectiva, las emociones se rigen por dos sistemas: el sistema de disposición individual, que se relaciona con los hábitos y costumbres, y el sistema de vigilancia que, cuando se moviliza, provoca que las personas reflexionen sobre sus decisiones habituales y reconsideren otras opciones no conocidas. Mientras el primer sistema contiene la recolección de decisiones tomadas en el pasado -a las que la persona recurre para tomar nuevas decisiones frente a situaciones que le producen miedo o ansiedad-, el segundo se activa cuando la persona se enfrenta a algo que considera inesperado o nuevo, y este sistema se encarga de analizarlo. La divergencia entre uno y otro, según la teoría, es que el sistema de vigilancia impulsa a buscar más información para tomar una decisión política, mientras que el sistema de disposición individual promueve actuar en concordancia con los hábitos ya conocidos, 


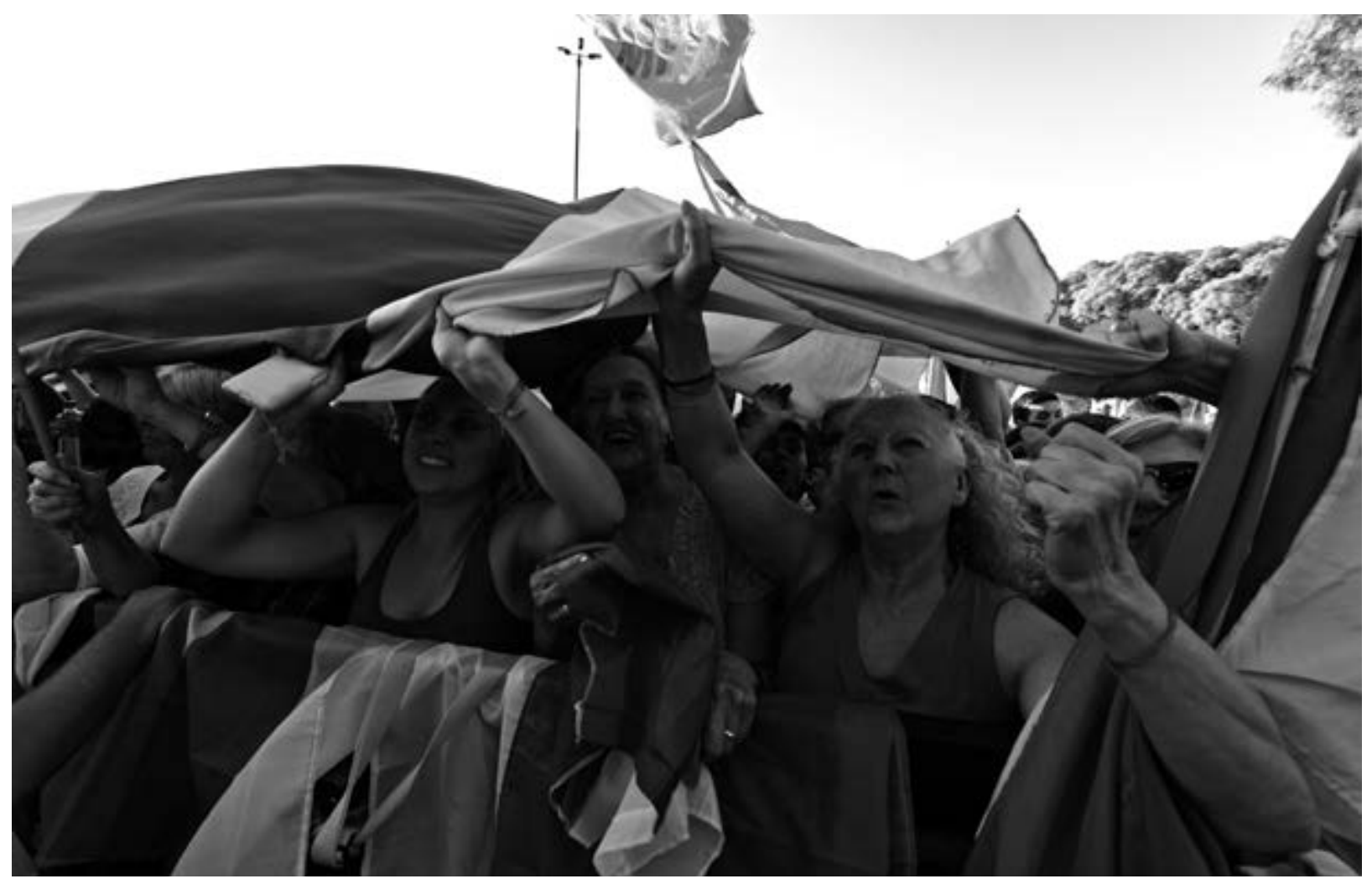

recurriendo a los marcos conceptuales que se concentran en el cerebro (Marcus, Neuman y Mackuen, 2000; Russell et al., 2007).

La activación de un sistema u otro depende de la información que se recibe. Cuando esa información coincide con las expectativas, se activa el sistema de disposición, impulsado por emociones positivas tales como el orgullo y la esperanza. En cambio, el sistema de vigilancia se activa cuando no coinciden las expectativas con la información recibida. Así, surgen como corolario la tristeza, la depresión, el enojo y la frustración. El entusiasmo o su ausencia, dicen los autores referidos, es la clave para comprender los componentes afectivos y cómo los individuos se conducen en sus decisiones políticas.

En el caso de una campaña política, la teoría de la inteligencia afectiva sugiere dos escenarios posibles. Si un candidato activa en los seguidores y electores el sistema de disposición - por apelar a emociones tales como el entusiasmo y la esperanza-, estos buscarán en sus hábitos, conductas y preferencias preexistentes, es decir, en sus marcos conceptuales ubicados en las redes del cerebro, para decidir qué pensar, cómo pensar y qué hacer respecto de ese candidato. Por el contrario, si el candidato activa en los votantes su sistema de vigilancia - por apelar a emociones tales como ansiedad, preocupación, ira, frustración-, estimulará a que estos presten atención al origen de la amenaza, con el fin de informarse y obtener un mejor conocimiento de la situación y de las causas de esa amenaza. El sistema de vigilancia favorece que el votante abandone sus predisposiciones y busque información nueva y original. De esta manera, las emociones condicionan qué camino seguir: mantener los hábitos políticos o buscar nueva información que pone en duda y en riesgo los hábitos, conductas y creencias preexistentes (Marcus et al., 2000; Russell et al., 2007).

El modelo de la inteligencia afectiva predice una relación directa entre las emociones evocadas y la atención que se presta a las noticias en las campañas. El sistema de vigilancia, relacionado con las emociones de miedo y ansiedad, incrementa la atención prestada a la información que proveen los medios para saber cómo actuar (Valentino, Hutchings, Banks y Davis, 2008; Valenzuela, 2011). Por su parte, el sistema de disposición, movilizado por el entusiasmo, se activa cuando se persigue un fin y la persona tiene esperanza de poder alcanzarlo. Tanto el miedo como el entusiasmo son las emociones que llevan a la movilización social y a la participación política (Castells, 2012).
Foto: ${ }^{\odot A F P / ~}$

Pablo Porciúncula. Simpatizantes del Frente Amplio durante el acto de cierre de campaña de Tabaré Vázquez en Montevideo, Uruguay, noviembre de 2014 
La apelación a emociones por parte de los dirigentes políticos estimula a los votantes a involucrarse en la participación y en procesos de comunicación como las interacciones en redes sociales. Las personas que sienten emociones, ya sean positivas o negativas, son más propensas a prestar atención a los mensajes de las campañas políticas, y a intercambiar información y opiniones, que aquellas que experimentan apatía o ausencia de emociones. Es decir que los mensajes que apelan a la emoción, además de ser más y mejor recordados, generan contagio emocional y se difunden con más rapidez que los contenidos donde se privilegia el razonamiento y la cognición (Hatfield, Cacioppo y Apson, 1994; Lang, 1994).

Las emociones, positivas o negativas, afectan el modo en que la información es decodificada (Lang, 2000). En otras palabras: las emociones ayudan a la gente a recordar los mensajes políticos. El miedo, la ansiedad o la ira permiten a los candidatos enfatizar valores consensuados -los marcos conceptuales de los que habla Lakoff- que facilitan la movilización de los electores. Asimismo, el uso de apelaciones emocionales sería consistente con la preferencia de los medios por el drama y la excitación, recursos narrativos fundamentales. Es justamente la narración el mecanismo que activa las redes cerebrales donde se alojan los marcos conceptuales. Así que se puede afirmar, junto con Brader (2006), que estos recursos dominan el paisaje retórico de las campañas (Amado, 2013; Bennett, 2012).

Estudios recientes han determinado que los spots actuales tienen un alto contenido emocional, que apela en particular a emociones como el orgullo, la seguridad, la confianza y la esperanza (Aruguete y Riorda, 2014; García Beaudoux, D’Adamo y Slavinsky, 2011). Los candidatos diseñan sus mensajes para generar entusiasmo en sus simpatizantes, y miedo y ansiedad en sus oponentes (Brader, 2005).

\section{La emoción en la comunicación política}

Valenzuela -quien ha empleado la teoría de la inteligencia afectiva para estudiar la conducta de los electores de Estados Unidos en 2011- sostiene que, a pesar de que el campo de la comunicación política ha sumado estudios donde las emociones cumplen un rol central (Brader, 2005; Coleman y Wu, 2010; Valentino et al., 2008), la investigación se ha fundado sobre la cognición y la razón, en el entendido de que las emociones no son centrales a la experiencia humana (Valenzuela, 2011). Las apelaciones a la emoción, afirma este autor, intentan despertar una respuesta emocional en los destinatarios, ya sea positiva o negativa, que se espera influya en sus decisiones políticas. Para evocar emociones negativas se usan mensajes que generen miedo, enojo, ira, angustia y frustración en los destinatarios. En cambio, para promocionar emociones positivas se diseñan mensajes que provoquen sentimientos de gratitud, seguridad, esperanza y orgullo (Valenzuela, 2011).

En la comunicación política el miedo y la esperanza parecen ser caras de una misma moneda: si la esperanza es el anhelo de cumplir determinado objetivo, el miedo a no concretarlo viene a ser su contraparte (Just, Crigler y Belt, 2007). Como la mente humana tiene la posibilidad de imaginar el futuro como característica esencial, la esperanza aparece como un recurso propicio para activar los mapas cerebrales que promueven el comportamiento político (Castells, 2008). Sin embargo, la esperanza está acompañada del miedo; el miedo al oponente. El entusiasmo es distinto a la esperanza (Castells, 2008), porque el entusiasmo es presente y pasajero. La esperanza es la mirada al futuro, que se acompaña de la promesa de llegar a un objetivo. En el caso del entusiasmo, que puede confundirse con esperanza, no se encuentra el anhelo, el deseo del cambio social.

Ted Brader también ha estudiado el vínculo entre la apelación al entusiasmo y al miedo en piezas de campaña, así como el compromiso de los ciudadanos 


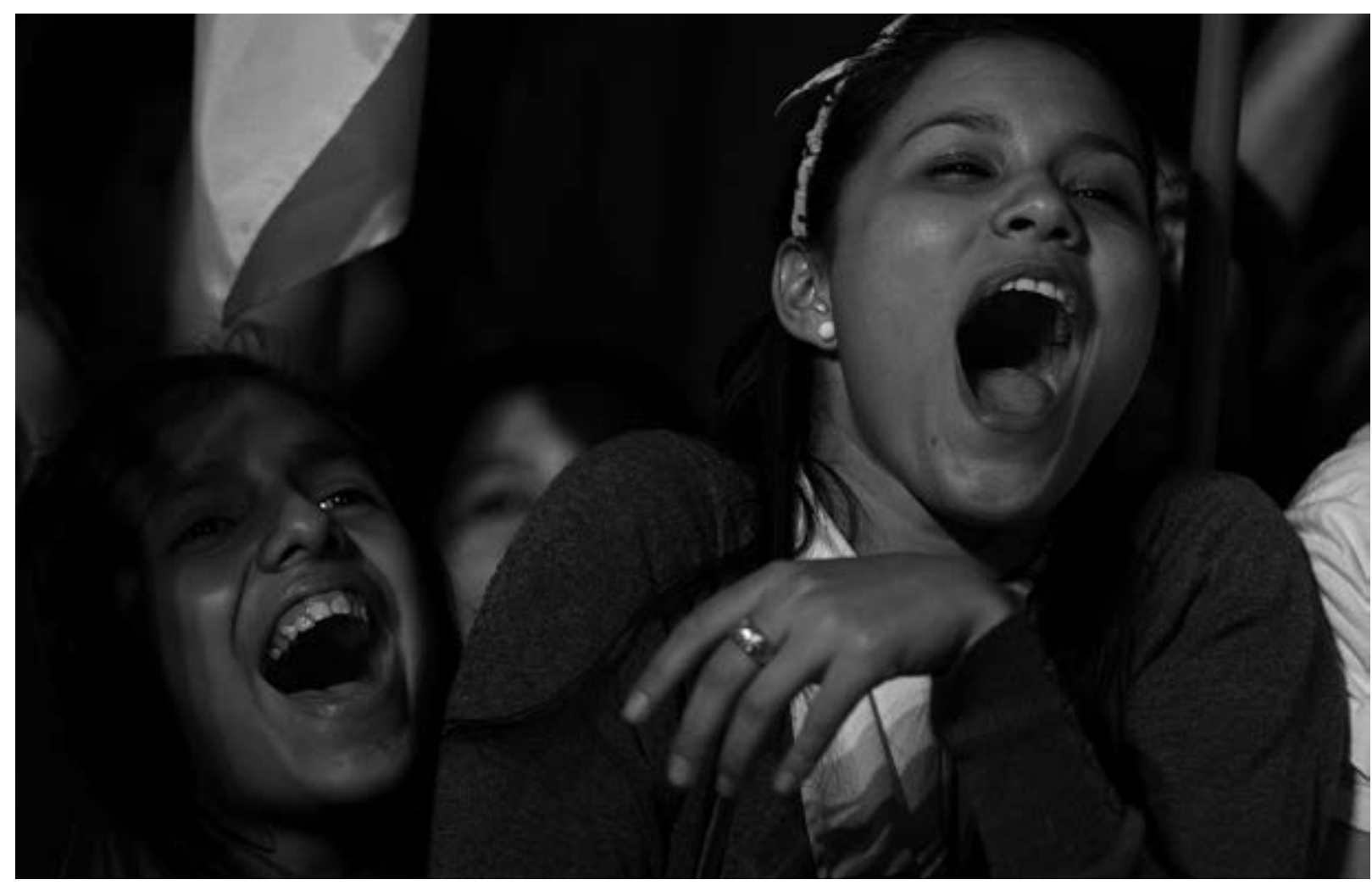

destinatarios con las cuestiones públicas, para probar empíricamente el papel de las emociones en la comunicación política. Su caso de estudio se sitúa en Estados Unidos, con una muestra de votantes que participaban de las elecciones primarias para gobernador en el estado de Massachusetts. En su investigación - pionera en el uso de los marcos analíticos de la teoría de la inteligencia afectiva- analiza si los dirigentes políticos y sus colaboradores de campaña hacen un uso estratégico de las emociones en los avisos televisivos, en el contexto electoral. Según concluye el estudio, los avisos que apelan a la esperanza y al entusiasmo motivan a los destinatarios a participar en la campaña. Por el contrario, los anuncios que despiertan miedo motivan a reconsiderar las antiguas preferencias políticas y a reflexionar sobre las decisiones, buscando más información para obtener seguridad (Brader, 2005). Brader demuestra que el entusiasmo activa a los votantes, a la vez que polariza sus opciones, al reafirmar y hasta incrementar el rechazo hacia el dirigente político opositor. La investigación prueba empíricamente que la emoción no sustituye a la razón, sino que activa el análisis de información en el proceso de toma de decisiones (Castells, 2008).

La emoción y la información están relacionadas. El contenido y los argumentos a menudo deben transmitir un mensaje general. [...] El mensaje ha de indicar a los votantes qué les debe causar miedo o esperanza y, en muchos casos, lo que los votantes deben hacer con dichos sentimientos... Las emociones no son una mera extensión del argumento. Confieren fuerza al argumento, no tanto por hacerlo más conveniente como por reorientar la atención y motivar al pensamiento a actuar. Las emociones nos envían señales para decir: “¡Eso es importante!”. Y la rapidez de nuestras respuestas emocionales permite que este proceso sesgue cómo interpretamos la información que recibimos, para bien o para mal (Brader, 2006, p. 185).

Si bien para su estudio Brader se concentra en la utilización estratégica de las emociones - a partir del uso de la música, las tomas de las cámaras y los protagonistas presentes en las imágenes- en los avisos televisivos de campaña, la influencia de las emociones en la decodificación no solo ha sido abordada desde los mensajes audiovisuales. La importancia del lenguaje en la activación de los marcos conceptuales ubicados en las redes cerebrales ha motivado también estudios sobre piezas comunicacionales escritas. De esta manera, las investigaciones previas indican que el estímulo a la emoción, tanto en términos de palabras emotivas como en un encuadre emotivo del mensaje, influye en el grado de atención que se le presta.
Foto: ${ }^{\oplus A F P} /$ Pablo Porciúncula. Seguidores del candidato presidencial Efrain Alegre en el acto de cierre de campaña en Asunción del Paraguay, abril de 2013 
A modo de conclusión

Los nuevos procesos comunicacionales en general, y en el caso de la comunicación política en particular, precisan nuevos encuadres teóricos para abordar su estudio. Es preciso buscar aproximaciones interdisciplinarias que permitan entender la complejidad de los novedosos formatos comunicacionales, las exigentes demandas de los ciudadanos y las constantes demandas de los dirigentes políticos que buscan comunicar, apelar, convencer a una audiencia cada vez más dispersa.

La comunicación política en los medios digitales se encuadra dentro de este escenario. Por ello, la teoría de la inteligencia afectiva puede dar luz a un tema que lleva pocos años de estudio, al menos en América Latina. Los medios digitales responden a una estructura donde priman las emociones. Desde los emoticones, que permiten comunicar las emociones que despierta una publicación, hasta la posibilidad de incluir fotos y videos: toda la arquitectura de los medios digitales revela la necesidad de estudiar los procesos comunicacionales que allí se producen mediante el uso de marcos analíticos interdisciplinarios. En este contexto, la teoría de la inteligencia afectiva aparece como una opción relevante y pertinente para su estudio.

\section{Referencias}

Amado, A. (2013). La épica no es fiesta [El drama como estilo de comunicación del gobierno argentino]. Perspectivas, 8, 2-6.

Aruguete, N. y Riorda, M. (2014). ¿ldeología u homogeneización? un análisis de las campañas electorales de Argentina, México y Venezuela. Revista Mexicana de Opinión Pública, 16, 31-49.

Banning, S. y Coleman, R. (2009). Louder than words. Visual Communication Quaterly, 16, 4-17.

Bennett, W. (2012). The Personalization of Politics: Political Identity, Social Media, and Changing Patterns of Participation. The ANNALS of the American Academy of Political and Social Science, 644(1), 20-39. http://doi. org/10.1177/0002716212451428

Bennett, W. L. (1998). The uncivic culture: Communication, identity, and the rise of lifestyle politics. P\&S: Political Science et Politics, 31(4), 740-761.
Bennett, W. L., e lyengar, S. (2008). A New Era of Minimal Effects? The Changing Foundations of Political Communication. Journal of Communication, 58(4), 707-731. http://doi.org/10.1111/j.14602466.2008.00410.x

Blumler, J. G., y Gurevitch, M. (1995). The Crisis of Public Communication [Versión Kindle]. London: Routledge.

Blumler, J. G., y Kavanagh, D. (1999). The Third Age of Political Communication: Influences and Features. Political Communication, 16(3), 209-230. http://doi.org/10.1080/105846099198596

Brader, T. (2005). Striking a responsive chord: How political ads motivate and persuade voters by appealing to emotions. American Journal of PoliticalScience, 48(2), 388-405. http://doi.org/10.1111/ j.0092-5853.2005.00130.x

Brader, T. (2006). Campaigning for Hearts and Minds, How Emotional Appeals in Political Ads Work. Chicago: University of Chicago.

Castells, M. (2008). Comunicación, poder y contrapoder en la sociedad red (I). Los medios y la política. Telos: Cuadernos de Comunicación e Innovación, 74, 13-24. Recuperado de http://dialnet.unirioja.es/ servlet/articulo?codigo $=2545714$

Castells, M. (2009). Comunicación y poder. Barcelona: Alianza.

Castells, M. (2012). Redes de indignación y esperanza. Los movimientos sociales en la era de internet. Barcelona: Alianza.

Chaffee, S. y Schleuder, J. (1986). Measurement and effects of attention to media news. Human Communication Research, 13 (1), 76-107.

Coleman, R. y Wu, H. D. (2010). Visual Cues in the Formation of Affect. Coleman, R. y Wu, H. D. (Eds.). Image and Emotion in Voter Decisions: The Affect Agenda. London: Lexington Books.

Dahlgren, P. (2012). Tracking the Civic Subject in the Media Landscape: Versions of the Democratic Ideal. Television \&t New Media, 14 (1), 71-88. http://doi.org/10.1177/1527476412458809

Damasio, A. (1994). Descartes's Error. New York: Avon Books. http:// doi.org/10.7202/051028ar

Damasio, A. (2007). En busca de Spinoza. España: Drakontos.

Dang-Xuan, L., Stieglitz, S., Wladarsch, J. y Neuberger, C. (2013). An Investigation of Influentials and the Role of Sentiment in Political Communication on Twitter During Election Periods. Information, Communication \& Society, 16(5), 795-825. http://doi.org/10.108 0/1369118X.2013.783608

Espinosa Pezzia, A. (2008). Decidiéndose por el mal menor: El rol de las emociones en las elecciones peruanas del 2006. Psicología Política, 37, 47-70. 
Eveland, W. (2004). The effect of political discussion in producing informed citizens: The roles of information, motivation, and elaboration. Political Communication, 21 (2), 177-193.

Garcia Beaudoux, V., D'Adamo, O. y Slavinsky, G. (2011). Propaganda gubernamental. Tácticas e iconografías del poder. Buenos Aires: La Crujía.

Gutiérrez-Rubi, A. (2009). Micropolítica: Ideas para cambiar la comunicación política. Recuperado de http://www.gutierrez-rubi.es/ wp-content/uploads/2010/01/libro_micropolitica.pdf

Hatfield, E., Cacioppo, J. y Apson, R. (1994). Emotional Contagion. New York: Cambridge University Press.

lyengar, S. y Kinder, D. (1987). News that matters: Television and American Opinion. Chicago: University of Chicago Press.

Jerit, J. (2004). Survival of the Fittest: Rethoric during the Course of an Election Cam- paign. Political Psicology, 25, 563-575.

Just, M., Crigler, A. y Belt, T. (2007). "Don't give up hope". Emotions, Candidate, Appraisal and Votes. Marcus, G. E., Russell, N. W., Mackuen, M. y Crigler, A. (Eds.). The affect effect, dynamics of emotion in political thinking and behavior (pp. 231-259). Chicago: University of Chicago Press.

Katz, E. y Lazarsfeld, P. (1955). Personal influence. Nueva York: The Free Press.

Lakoff, G. (2007). No pienses en un elefante:lenguaje y debate político. Madrid: Editorial Complutense.

Lang, A. (1994). How they predict memory for Television Messages. Presentado en el Annual Meeting of the Association for Education in Journalism and Mass Communication. Atlanta, Estados Unidos.

Lang, A. (2000). The limited capacity model of mediated message processing. Journal of Communication, 50, 46-70. http://doi. org/10.1111/j.1460-2466.2000.tb02833.x

LeDoux, J. (1996). The Emotional Brain. London: Phoenix.

López-Escobar, E. y Llamas, J. P. (1996). Agenda-setting: investigaciones sobre el primer y el segundo nivel. Comunicación y Sociedad, 9 (2), 9-15.

Marcus, G. E. (2002). The Sentimental Citizen, Emotion in Democratic Politics. Pennsylvania: Pennsylvania State University Press.

Marcus, G., Neuman, R. y Mackuen, M. (2000). Affective Intelligence and Political Judgment. Chicago: The University of Chicago Press.

McCombs, M. y Shaw, D. (1974, agosto). A Progress Report on Agenda-setting Research. Paper presentado en la reunión anual de la Association for Education in Journalism, San Diego, CA.
Miller, J. M. (2007). Examining the Mediators of Agenda-Setting: A New Experimental Paradigm Reveals the Role of Emotions. International Society of Political Psychology, 28(6), 689-717.

Moy, P. y Gastil, J. (2006). Predicting Deliberative Conversation: The Impact of Discussion Networks, Media Use, and Political Cognitions. Political Communication, 23(4), 443-460. http://doi. org/10.1080/10584600600977003

Plummer, K. (2003). Intimate Citizenship. Seattle: University of Washington Press.

Reese, S. D. (2001). Framing Public Life: A Bridging Model for Media Research. Reese, S., Gandy, 0. y Grant, A. (Eds.). Framing Public Life: A Bridging Model for Media Research (pp. 7-31). Mahwah, New Jersey: Lawrence Erlbaum.

Rincón, 0. (2011). Mucho ciberactivismo... pocos votos. Nueva Sociedad, 235, 74-89. Recuperado de http://library.fes.de/pdf-files/ nuso/nuso-235.pdf

Rincón, O., y Bonilla, J. I. (2004). Comunicación Política en América Latina. Bogotá: Centro de Competencia en Comunicación para América Latina. Recuperado de http://www.fesmedia-latin-america.org/uploads/media/Comunicaci\%C3\%B3n_pol\%C3\%ADtica_en_Am\%C3\%A9rica_Latina.pdf

Russell, N. W., Marcus, G., Crigler, A. y Mackuen, M. (2007). Affect Effect. Dynamics of emotion in polititical thinking and behaviour. Chicago: University of Chicago.

Scherer, K. R. (2005). What are emotions? And how can they be measured? Social Science Information, 44(4), 695-729. http:// doi.org/10.1177/0539018405058216

Stieglitz, S. y Dang-Xuan, L. (2013). Emotions and Information Diffusion in Social Media-Sentiment of Microblogs and Sharing Behavior. Journal of Management Information Systems, 29(4), 217-248. http://doi.org/10.2753/MIS0742-1222290408

Valentino, N., Hutchings, T., Banks, A. y Davis, A. (2008). Is a worried citizen a good citizen? Emotions, political information seeking, and learning via the internet. Political Psicology, 29 (2), 247-273.

Valenzuela, S. (2011). The Affective Citizen Communication Model: How Emotions Engage Citizens with Politics through Media and Discussion. ProQuest Dissertations and Theses, 198. Recuperado de http://search.proquest.com/docview/906381170

Westen, D. (2008). The emotional brain. New York: Public Affairs. Wolton, D. (2005). Elogio del gran público. Buenos Aires: Gedisa.

DOI: http://dx.doi.org/10.22235/d.v0i25.1271 